\title{
Optimum IMFs Selection Based Envelope Analysis of Bearing Fault Diagnosis in Plunger Pump
}

\author{
Wenliao Du, ${ }^{1}$ Zhiyang Wang, ${ }^{2}$ Xiaoyun Gong, ${ }^{1}$ Liangwen Wang, ${ }^{1}$ and Guofu Luo' \\ ${ }^{1}$ School of Mechanical and Electronic Engineering, Zhengzhou University of Light Industry, Zhengzhou 450002, China \\ ${ }^{2}$ School of Mechanical and Power Engineering, Henan Polytechnic University, Jiaozuo 454000, China \\ Correspondence should be addressed to Wenliao Du; dwenliao@zzuli.edu.cn
}

Received 9 June 2015; Accepted 7 September 2015

Academic Editor: Laurent Mevel

Copyright (c) 2016 Wenliao Du et al. This is an open access article distributed under the Creative Commons Attribution License, which permits unrestricted use, distribution, and reproduction in any medium, provided the original work is properly cited.

\begin{abstract}
As the plunger pump always works in a complicated environment and the hydraulic cycle has an intrinsic fluid-structure interaction character, the fault information is submerged in the noise and the disturbance impact signals. For the fault diagnosis of the bearings in plunger pump, an optimum intrinsic mode functions (IMFs) selection based envelope analysis was proposed. Firstly, the WignerVille distribution was calculated for the acquired vibration signals, and the resonance frequency brought on by fault was obtained. Secondly, the empirical mode decomposition (EMD) was employed for the vibration signal, and the optimum IMFs and the filter bandwidth were selected according to the Wigner-Ville distribution. Finally, the envelope analysis was utilized for the selected IMFs filtered by the band pass filter, and the fault type was recognized by compared with the bearing character frequencies. For the two modes, inner race fault and compound fault in the inner race and roller of rolling element bearing in plunger pump, the experiments show that a promising result is achieved.
\end{abstract}

\section{Introduction}

The plunger pump constitutes the key part in hydraulic cycle, which is widely used in the huge construction machinery, marine engine, military equipment, and so forth. The pump performance directly relates to the reliability of the whole hydraulic cycle, so the condition monitoring and fault diagnosis on the pump is very important [1]. In the pump, the bearing supports the main shaft, and it is one of the easiest infection components. The spectrum analysis on the bearing vibration signal, and further deducing the fault type, is a main fault diagnosis approach for the bearing of plunger pump $[1,2]$.

Bearing vibration signal exhibits a typical amplitude modulation character, and the envelope analysis is a powerful tool to obtain the bearing fault characteristic frequency. In order to overcome the effect of noise and other extraneous information, a band pass filter with an appropriate central frequency is first worked on the signal. In tradition, the Fourier transform (FT) is utilized to obtain the resonance frequency band first. Then, the frequency band is selected to construct the band pass filter, and the envelope demodulation is finally used to acquire the fault character. As the vibration signal of bearing in plunger pump shows a typical nonstationary character, the Fourier transform is not appropriate in this situation. The short time Fourier transform (STFT) is an effective approach for the nonstationary signal. Pan and Tsao [3] utilized the STFT to obtain the energy spectrum, and then the resonance frequency band is decided. In addition, Liu et al. [4] obtained the amplitude spectrum entropy of each frequency band through the STFT, and then the envelope analysis is employed to determine the bearing fault type. As reported by Jiang et al. [5], the time-frequency resolution ratio is invariant for STFT, which is a limitation for the signal with strong noise level. The Wigner-Ville distribution (WVD) is independent of the window size and type, and it has a two-order time-frequency distribution with energy explanation and a superior time-frequency resolution ratio. Though the WVD usually suffers from the disturbance of cross terms, the pseudo Wigner-Ville distribution (PWVD) 
offers an alternative selection. A plenty of examples show that the PWVD has good time-frequency centralization and is widely used in mechanical fault diagnosis [6].

To further overcome the unfavorable effect of redundant frequency components in the signal, the necessary data transformation on the signal is utilized to acquire the fault characteristic frequency band. The empirical mode decomposition (EMD) is a self-adaptive signal analysis method, which makes it more superior to other multiresolution approaches, such as STFT and wavelet transform (WT), in extracting the characteristic frequency bands. In general, the number of intrinsic mode functions (IMFs) got from the EMD is far more than the number of independent components in the signal. So, to select the most relevant IMFs to the fault from these IMFs is serious and necessary for improving the diagnostic accuracy. Aiming at this issue, a variety of methods were presented. Peng et al. selected the appropriate IMFs depending on the measure factor based on distance [7], and an optimal band pass filter was constructed with the central frequency and bandwidth generated using the fast kurtogram. Peng et al. [8], Wang et al. [9], and Guo and Tse [10] utilized the correlation analysis between the IMF and the original signal to select the candidate IMFs. In addition, Boutana et al. [11] proposed an IMFs selection method based on the Minkowski distance and Jensen Rényi divergence of order $\alpha$. The above mentioned methods are intuitive and feasible, but they all neglected the a priori fault knowledge of the diagnosis object, and the threshold is determined empirically. For the gear box fault diagnosis, Wang and Heyns [12] selected the appropriate IMFs based on the gear meshing frequency. But, in the high frequency resonant system, this a priori knowledge cannot be used directly.

The bearing fault diagnosis is a principal content to insure the plunger pump operating reliably. As the pump always works in a high speed and heavy load situation and the pump self is a typical mechanical-hydraulic coupling system, the collected vibration signal shows strong nonstationary and nonlinear characteristic [13], and the bearing character signals are always submerged in the noise and the disturbance impact signals coming from plungers and swash plate, which makes it more difficult to extract the effective diagnosis information. In this paper, the Wigner-Ville distribution is used to extract the bearing resonance frequency band first. Then, the EMD procedure is performed to the vibration signal, and the appropriate IMFs are selected based on the resonance frequency band, and the filter bandwidth is determined. Finally, the envelope analysis is utilized to the filtered signal to implement the bearing fault diagnosis in plunger pump.

\section{Optimum IMFs Selection Based Procedure to Detect Bearing Faults}

2.1. Wigner-Ville Distribution. The Wigner-Ville distribution can be regarded as a special case of Cohen's class [14]. This distribution is a Fourier transform for the instantaneous autocorrelation function of signal, and the result reflects the time-frequency character of the signal. For a signal $x(t)$, its WVD is defined as

$$
\begin{aligned}
& \operatorname{WVD}_{x}(t, \omega) \\
& \quad=\frac{1}{2 \pi} \int_{-\infty}^{+\infty} x^{*}\left(t-\frac{1}{2} \tau\right) x\left(t+\frac{1}{2} \tau\right) e^{-i \tau \omega} d \tau,
\end{aligned}
$$

where $x^{*}$ is the complex conjugate of $x(t)$. For the discrete version of a signal, the WVD is defined as

$$
\operatorname{WVD}(n, k)=\sum_{q=-N}^{N-1} R[n, q] \cdot e^{-j 2 \pi k q / N},
$$

where $N$ is the number of sampling points, $q$ is the delay factor, and $R[n, q]$ is the instantaneous autocorrelation function of $x(t)$, which is determined by

$$
R[n, q]=x\left[n+\frac{q}{2}\right] \cdot x^{*}\left[n-\frac{q}{2}\right] .
$$

The WVD has some good characters, such as real valued, time-frequency marginal properties, time- and frequencycovariant, time- and frequency-dilation covariance, energy conservation. But, from formula (1) and formula (2), at a moment, if the signal has overlapping between the future and the past, even if the signal value at this moment is zero, the corresponding WVD is not zero, which is the cross terms problem in the WVD. To overcome this disturbance, both the temporal moving window and the frequency moving window are adopted to restrain the cross terms. This method is noted as smoothed pseudo Wigner-Ville distribution (SPWVD), which is defined as

$$
\begin{gathered}
\operatorname{SPW}_{x}(t, \omega)=\frac{1}{2 \pi} \int_{-\infty}^{+\infty} \int_{-\infty}^{+\infty} h(\tau) g(s-\tau) x^{*}\left(s-\frac{1}{2} \tau\right) \\
\cdot x\left(s+\frac{1}{2} \tau\right) e^{-i \tau \omega} d s d \tau,
\end{gathered}
$$

where $h$ and $g$ are window functions, which are used to restrain the cross terms in the time domain and frequency domain, respectively, and usually selected as Gauss function. Though the time-frequency resolution is less severely affected for this technology, it is widely used in the mechanical fault diagnosis $[6,14]$.

2.2. EMD Decomposition. The EMD was first reported by Huang et al. [15] in America as an adaptive time-frequency processing algorithm for a time series. It supposes that a signal is composites of a sequence of intrinsic mode functions (IMFs) and a residue with a monotonic pattern. Meanwhile, these IMFs are nearly orthogonal functions and satisfy the following two conditions: (1) In the entire data set, the number of local extrema and the number of zero crossings must be either equal or different by one at most. (2) At any point, the mean of the envelope defined by the local maxima and the envelope defined by the local minima must be zero. So, after the EMD processing, the obtained IMFs are almost monofrequency components. 
Given an original time series $x(t)$, through the decomposition procedure of EMD, it can be expressed as

$$
x(t)=\sum_{i=1}^{n} c_{i}+r_{n},
$$

where $r(t)$ is the residue of the signal, and it describes the mean trend of the signal or a constant. The IMFs $c_{1}, c_{2}, \ldots, c_{n}$ correspond to the components from high frequency to low frequency separating from the original signal.

2.3. Envelope Analysis. When the bearing has a fault, the fault information in the vibration signal always emerges in the form of modulation. The envelope analysis has been successfully applied to extract the periodical impact in the vibration signal. Usually, the band pass filtering process is first worked on the signal to enhance the fault character, and then the envelope analysis is used to obtain the fault frequency character in the low frequency. Several approaches have been reported to extract the envelope signal. In this paper, the widely used Hilbert transform based envelope extraction method is adopted [1]. For the signal $x(t)$, its Hilbert transform is defined as

$$
\widehat{x}(t)=\frac{1}{\pi} \int_{-\infty}^{+\infty} \frac{x(\tau)}{t-\tau} d \tau .
$$

So, the analytic signal corresponding to $x(t)$ is

$$
z(t)=x(t)+j \widehat{x}(t) .
$$

It can be expressed as a complex form

$$
z(t)=a(t) \exp (j \omega(t)),
$$

where $a(t)$ is the envelope signal, by taking the Fourier transform, the envelope spectrum of $x(t)$ is got. If the bearing has a fault, the fault characteristic frequency can be observed in the envelope spectrum.

\subsection{Optimum IMFs Envelope Spectrum Based Fault Diagnosis.} As the plunger pump has an inherent mechanical-hydraulic coupling character and the collected vibration signal contains a plenty of noise, usually the envelope analysis to the signal directly cannot get an accurate diagnosis result. In order to improve the performance of the envelope analysis further, combining with the prior knowledge of bearing faults, a Wigner-Ville distribution based optimum IMFs envelope spectrum fault diagnosis method is proposed in this paper, and the flow chart is plotted in Figure 1. The detailed instructions for this procedure are as follows.

Step 1. Use the SPWVD on the signal to obtain the timefrequency spectrum.

Step 2. Based on the a priori knowledge of fault frequency, select the band width with a higher resonance frequency as resonance frequency band.

Step 3. Use the EMD on the signal to obtain a series of IMFs.

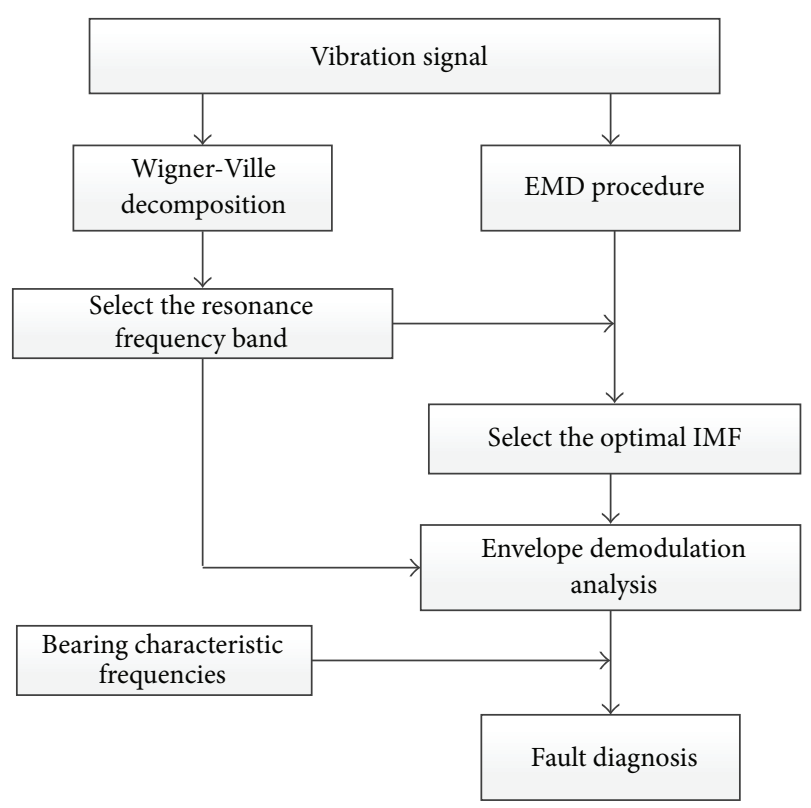

FIGURE 1: Flow chart of fault diagnosis.

Step 4. Based on the frequency range limited by the resonance frequency band, select the optimal IMF in the frequency scope.

Step 5. Reconstruct the selected IMF, and the band pass filter is employed to extract the useful information from the reconstructed signal with the resonance frequency band. Then, the envelope analysis is used to obtain the envelope spectrum.

Step 6. Compare the bearing characteristic frequencies with the envelope spectrum, and the bearing fault type is determined.

\section{Fault Diagnosis Experiment of Bearing in Plunger Pump}

For the inner race fault and compound fault in the inner race and roller of the big end bearing in plunger pump, in this section, the traditional envelope analysis and the proposed method are compared. The pump type is A11VLO190, which has nine plungers, and the bearing geometry is listed in Table 1. Single point faults are introduced to the test bearing using line-machining with fault diameters of $0.2 \mathrm{~mm}$, and the fault depth is $0.3 \mathrm{~mm}$. The position of the fault lies in the bearing inner race and roller to simulate the fault at different positions, respectively. The experimental rotating speed is about $1600 \mathrm{r} / \mathrm{min}$. An accelerometer is mounted on the bearing seat near the big end bearing of the pump shell to acquire the vibration signals from the bearing. The vibration data are acquired with a $10 \mathrm{kHz}$ sample frequency. Each bearing element has its own characteristic frequency of fault. Those frequencies can be calculated based on the kinematics relation. For a bearing with a stationary outer race, the characteristic bearing defect frequencies of inner race $\left(f_{i}\right)$ 


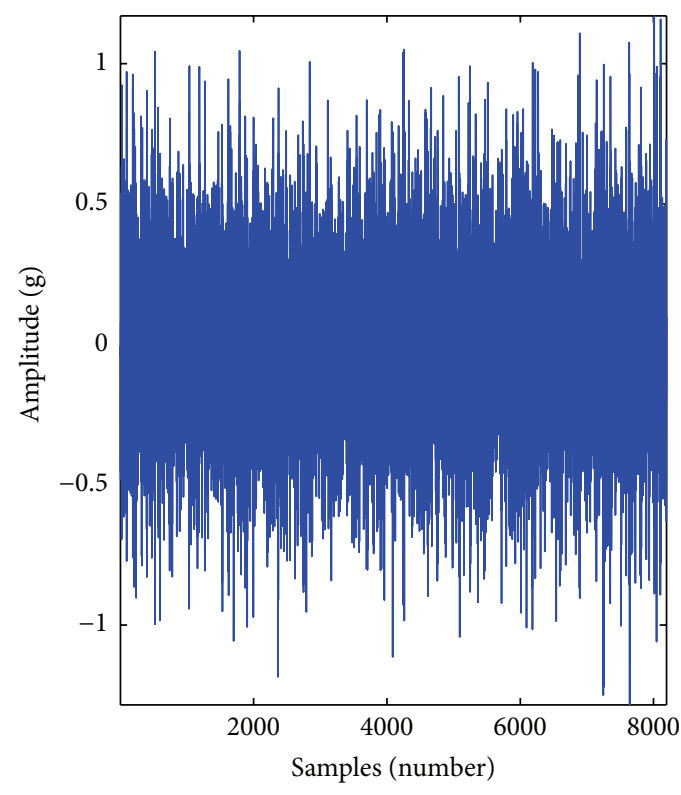

(a) Original vibration signal

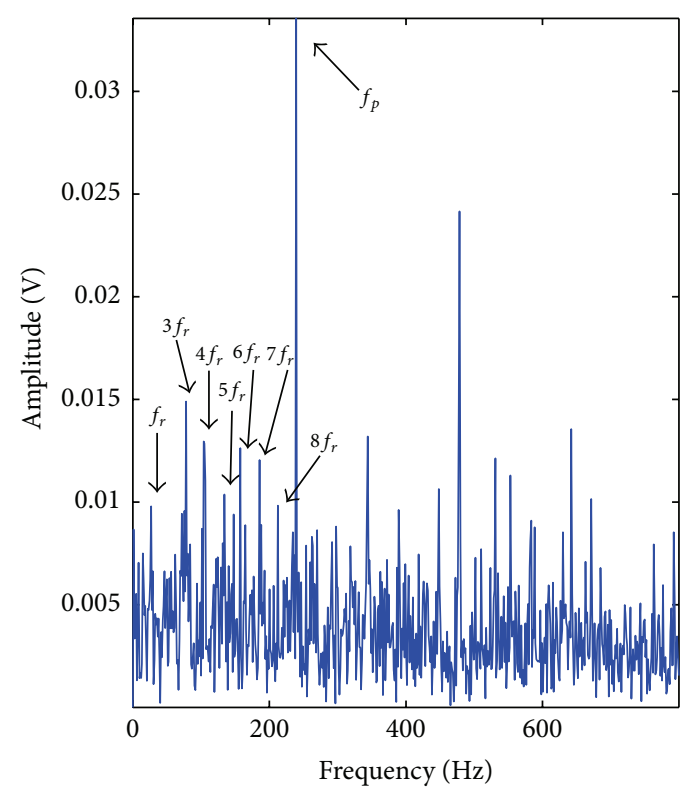

(b) Envelope spectrum

FIGURE 2: Original signal and envelope spectrum of bearing with inner race fault.

TABLE 1: Basic geometry information of the bearing.

\begin{tabular}{lccc}
\hline $\begin{array}{l}\text { Roller } \\
\text { number }\end{array}$ & $\begin{array}{c}\text { Roller diameter } \\
(\mathrm{mm})\end{array}$ & $\begin{array}{c}\text { Pitch diameter } \\
(\mathrm{mm})\end{array}$ & $\begin{array}{c}\text { Contact angle } \\
(\mathrm{rad})\end{array}$ \\
\hline 16 & 15 & 82 & 0 \\
\hline
\end{tabular}

TABLE 2: Characteristic frequencies of the bearing $(\mathrm{Hz})$.

\begin{tabular}{lcc}
\hline$f_{r}$ & $f_{i}$ & $f_{b}$ \\
\hline 26.7 & 252 & 70 \\
\hline
\end{tabular}

and roller $\left(f_{b}\right)$ are listed in Table 2, which are calculated using the following formula.

The characteristic bearing defect frequency of inner race $\left(f_{i}\right)$ :

$$
f_{i}=\frac{N_{b}}{2}\left[1+\left(\frac{B_{d}}{P_{d}}\right) \cos \alpha\right] f_{r}
$$

The characteristic bearing defect frequency of roller $\left(f_{b}\right)$ :

$$
f_{b}=\frac{1}{2}\left[\left(\frac{P_{d}}{B_{d}}\right)-\left(\frac{B_{d}}{P_{d}}\right) \cos ^{2} \alpha\right] f_{r}
$$

where $B_{d}$ is the ball diameter; $P_{d}$ is the pitch diameter; $N_{b}$ is the number of rollers; $\alpha$ is the contact angle; $f_{r}$ is the rotating frequency of the spindle.

The characteristic bearing defect frequencies of inner race and roller are $252 \mathrm{~Hz}$ and $70 \mathrm{~Hz}$, respectively, for the above bearings at the shaft rotational speed of $1600 \mathrm{r} / \mathrm{min}(26.7 \mathrm{~Hz}$ in axial frequency).
3.1. Bearing with Inner Race Fault. As the inner race is relatively far from the pump shell, the complicated signal transduction route results in extracting the characteristic information more difficult. Figure 2 shows the original signal and its envelope spectrum of the bearing vibration signal with inner race fault. In Figure 2(a), the original signal contains plenty of noise, and in its envelope spectrum in Figure 2(b), the axial frequency and the harmonic frequencies are very clear; especially the plunger impact frequency $f_{p}$ (nine times $f_{r}$ ) is more obvious. But, as affected by the noise, and the inner race fault information is extracted inherently difficultly, the inner race fault characteristic frequency is not observed. The Wigner-Ville distribution corresponding to Figure 2(a) is shown in Figure 3(a), which is evident that the bearing system resonance frequency bands are $1.2 \mathrm{kHz}-$ $1.5 \mathrm{kHz}$ and $3.3 \mathrm{kHz}-3.6 \mathrm{kHz}$, respectively. Decompose the original bearing signal with inner race fault through the EMD procedure, and get the first four IMFs and the corresponding envelope spectrum as shown in Figure 3(b). Compare with Figure 2(a), and select the resonance frequency band with a higher value for further analyze, which is $3.3 \mathrm{kHz}-3.6 \mathrm{kHz}$. Then, the envelope analysis is worked on this frequency band and obtains the envelope spectrum as Figure 3(c). In this figure, we can observe that the bearing inner race fault frequency is very clear, and the axial frequency, the harmonic frequencies, and the plunger impact frequency are still distinct.

3.2. Bearing with Compound Fault. In rolling bearings, besides the single fault, there is usually occurring compound fault. If a definite diagnosis and an appropriate maintenance cannot be carried out in time, a more serious disaster would not be avoided. For the compound fault, the complexity of 


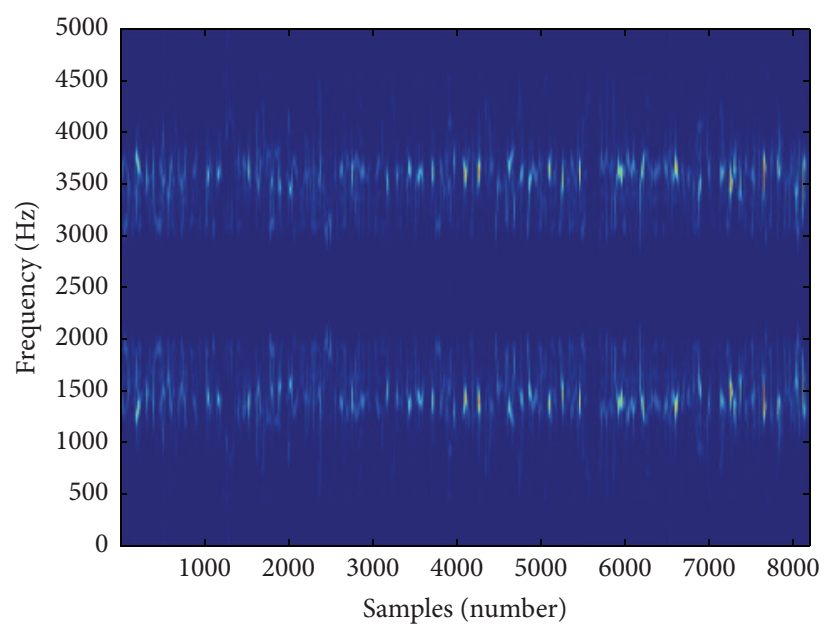

(a) Wigner-Ville distribution
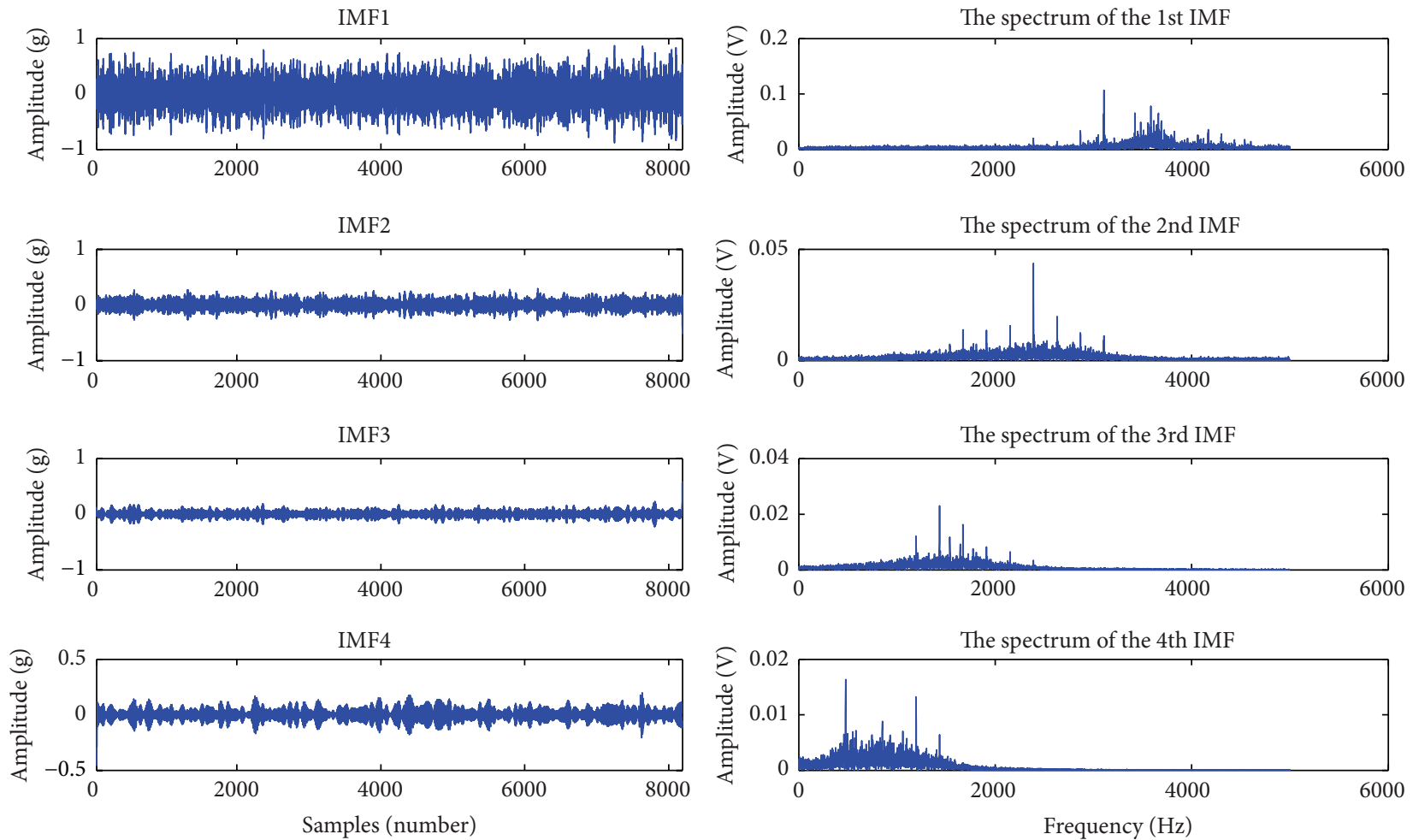

(b) The first four IMFs and their spectrums

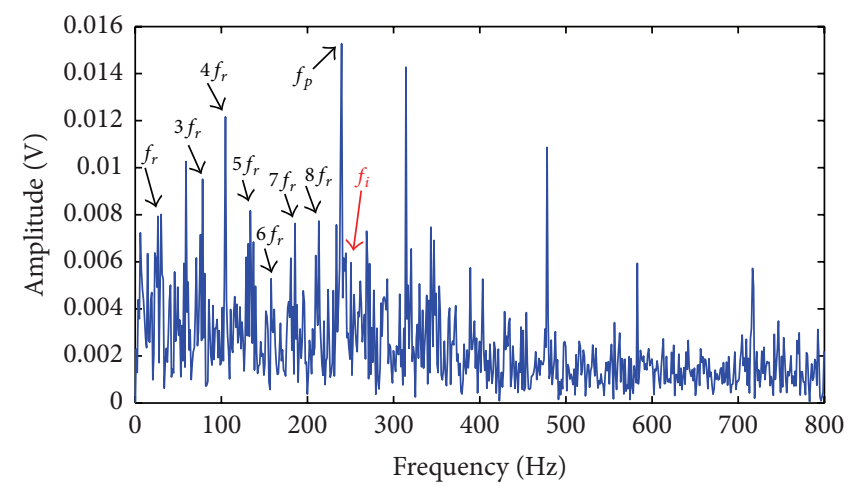

(c) Envelope spectrum of the optimum IMF

FIGURE 3: Bearing inner race fault diagnosis by envelope analysis for optimum IMF. 


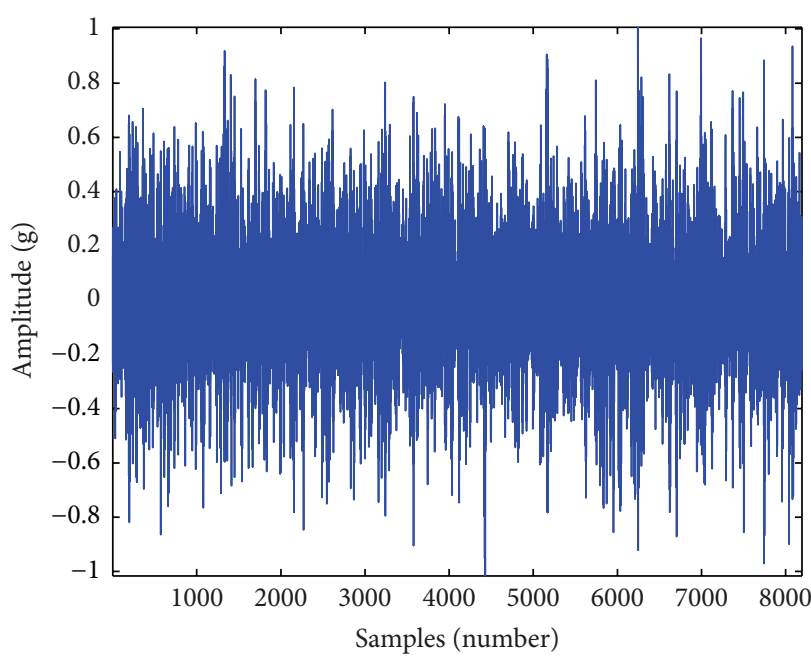

(a) Original vibration signal

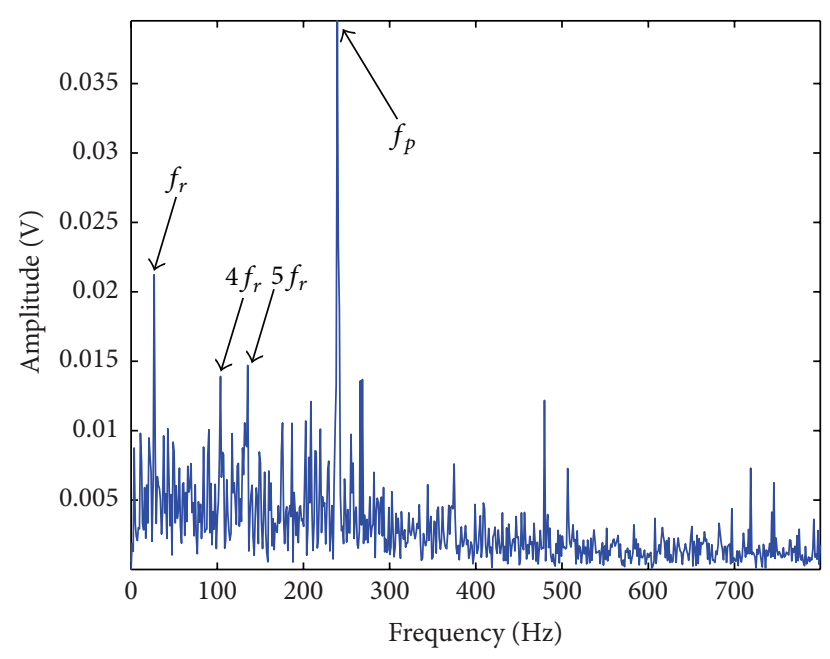

(b) Envelope spectrum

FIGURE 4: Original signal and envelope spectrum of bearing with compound fault.

every fault interrelated and interact on each other makes the fault diagnosis more challenging. Figure 4 shows the vibration signal and the corresponding envelope spectrum of bearing with the compound fault in the inner race and roller. It can be observed that the signal is contaminated by a tremendous amount of noise in Figure 4(a). In Figure 4(b), though the axial frequency, the harmonic frequencies of some orders, and the plunger impact frequency are very distinct, the inner race fault frequency and the roller characteristic frequency are concealed by the noise and other uncorrelated frequency components. From the Wigner-Ville distribution of the compound fault signal depicted in Figure 5(a), we can see the bearing system resonance frequency bands are $1.5 \mathrm{kHz}-2.0 \mathrm{kHz}$ and $3.1 \mathrm{kHz}-3.5 \mathrm{kHz}$. Decompose the signal through the EMD procedure, the first four IMFs, and the corresponding envelope spectrum as shown in Figure 5(b). Compare with Figure 5(a), the coincident frequency band is $3.3 \mathrm{kHz}-3.5 \mathrm{kHz}$. So, select the first IMF to reconstruct the signal and select the resonance frequency band $3.3 \mathrm{kHz}-3.5 \mathrm{kHz}$ to filter the obtained reconstruct time series. Then, the envelope analysis is employed to obtain the envelope spectrum as Figure 5(c). In this figure, the bearing inner race characteristic frequency and the roller characteristic frequency are both stand out, and the axial frequency, the harmonic frequencies are more vivid than in Figure 4(b), and the plunger impact frequency is still distinct.

By comparison, if an inappropriate IMF is selected, such as IMF3, the envelope spectrum is shown as Figure 6(a). Only the plunger impact frequency can be observed, and of course, the definite diagnosis cannot be achieved. In addition, if omitting the procedure of band pass filtering, the envelope analysis works directly on the IMF1, which is the optima IMF, and the obtained envelope spectrum is displayed in Figure 6(b). The axial frequency, some harmonic frequencies, the plunger impact frequency, and the inner race fault frequency are observed, but the roller fault cannot be detected.

\section{Conclusions}

(1) This study proposed an optimum IMFs selection with Wigner-Ville distribution based envelope analysis of bearing fault diagnosis for plunger pump, and it also can be used to other transmission elements. With the help of the adaptive time-frequency analysis of EMD and the selection of optima IMF through Wigner-Ville combining with the a priori fault knowledge, this method is more accurate and reliable compared with the traditional envelope analysis.

(2) For the single fault and compound fault of the big end bearing in plunger pump, the proposed method can restrain noise and interfering signal effectively and detect the bearing fault correctly.

(3) As using the a priori fault knowledge, compared with the result of traditional envelope analysis and the result of omitting the IMF selection procedure, the proposed method gets a more promising result.

\section{Conflict of Interests}

The authors declare that there is no conflict of interests regarding the publication of this paper.

\section{Acknowledgments}

This work is supported by the National Nature Science Foundation of China (Grants nos. 51205371, U1304523, and 51405453), the National Key Technology Research and Development Program of China (Grants nos. 2015BAF32B04 and 2012BAF12B13), the Key Scientific and Technological Project 


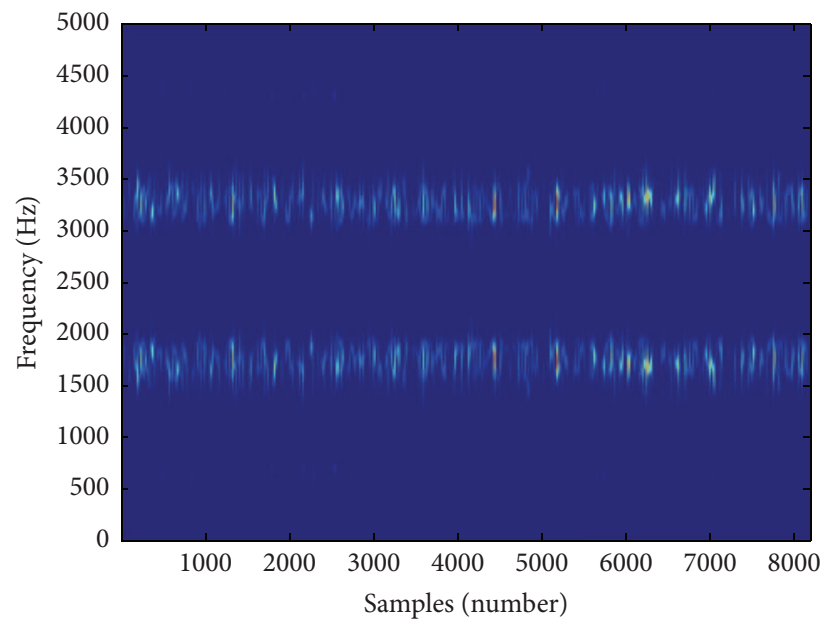

(a) Wigner-Ville distribution
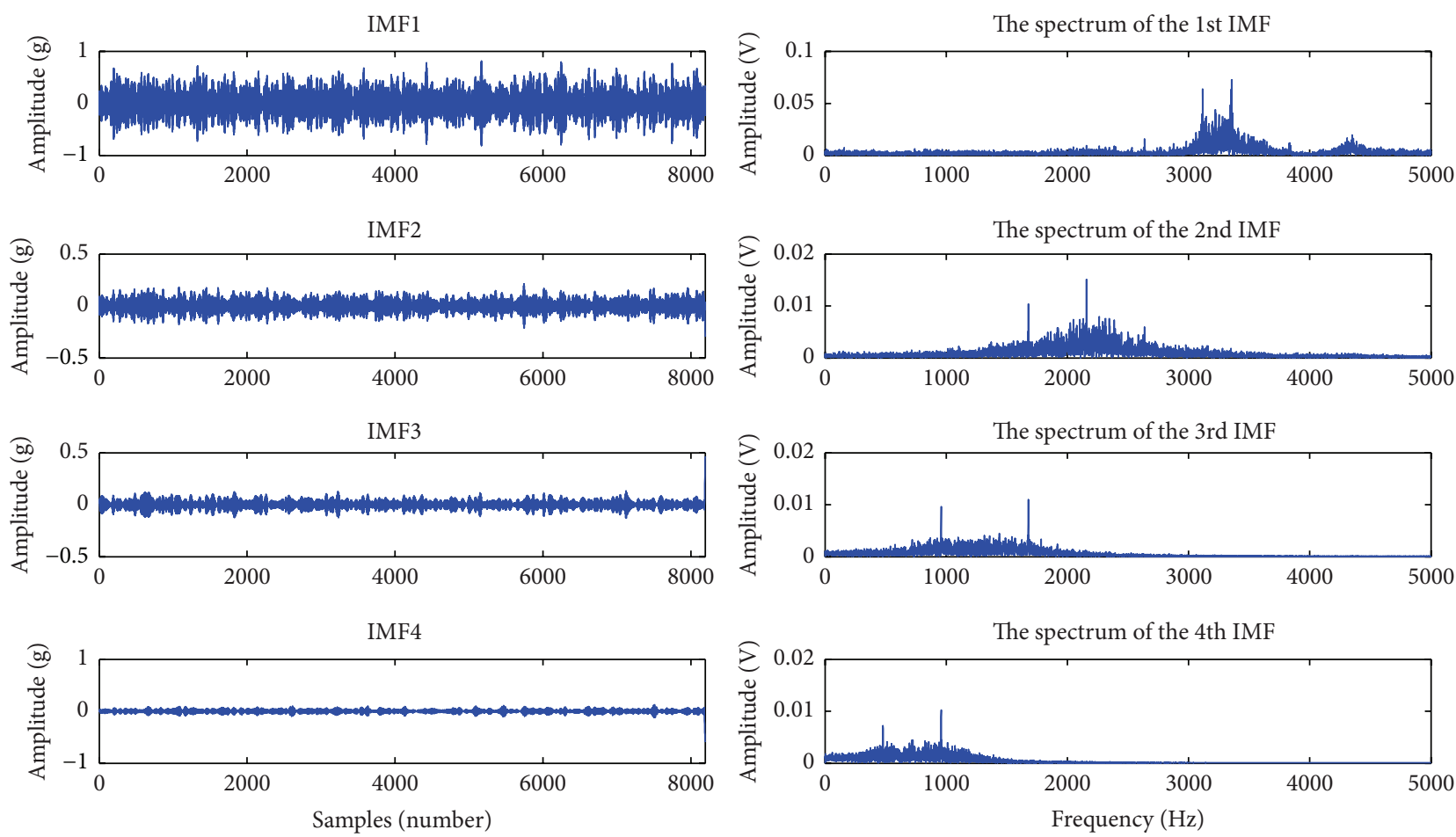

(b) The first four IMFs and their spectrums

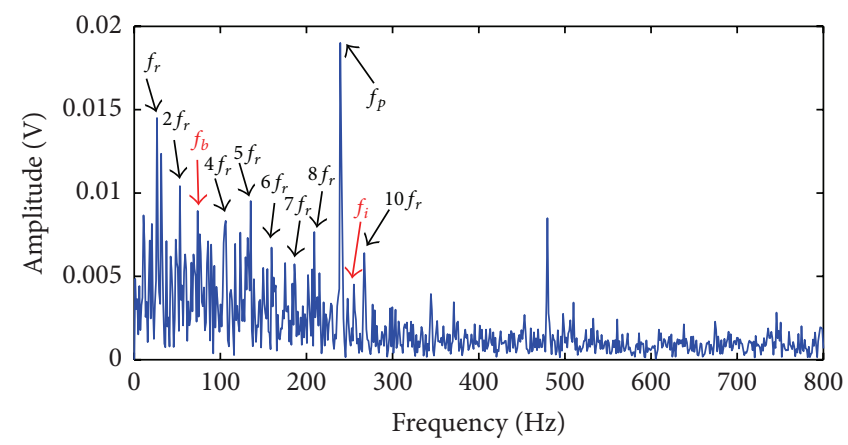

(c) Envelope spectrum of the optimum IMF

FIGURE 5: Bearing compound fault diagnosis by envelope analysis for optimum IMF. 


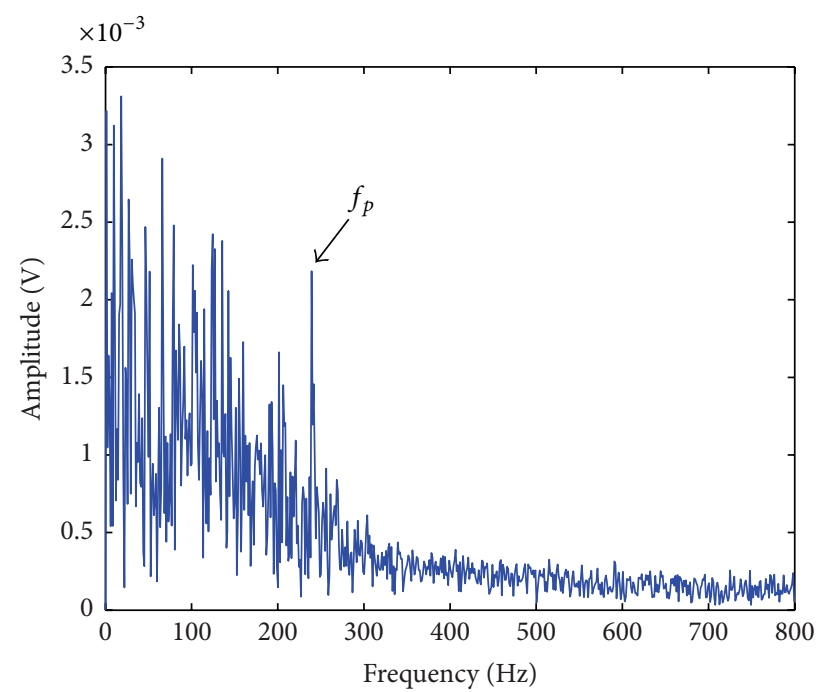

(a) Envelope spectrum corresponding to IMF3

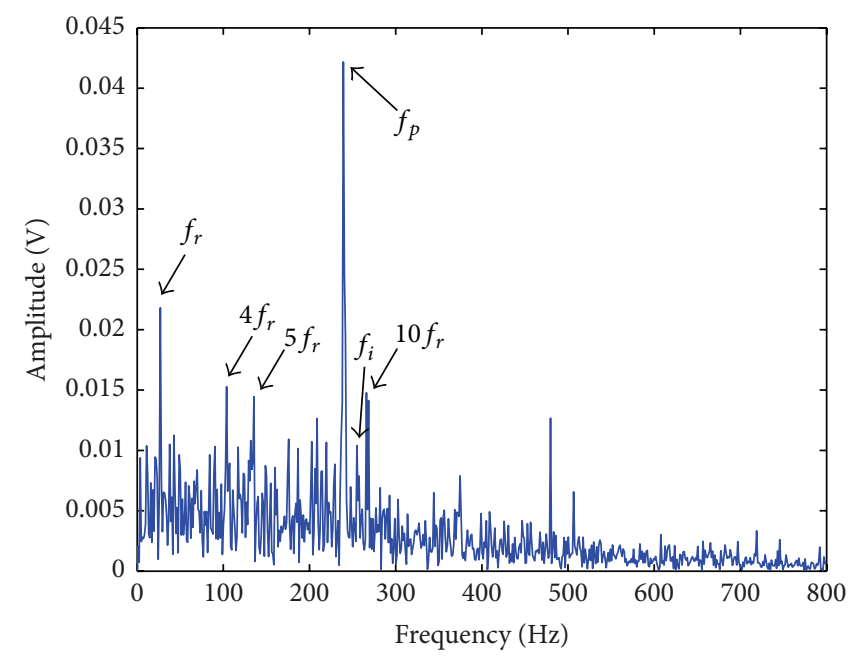

(b) Envelope spectrum corresponding to IMF1

FIGURE 6: Bearing compound fault diagnosis by traditional procedures.

of Zhengzhou City of China (Grant no. 131PPTGG411-2), and the Doctoral Starting-Up Foundation of Zhengzhou University of Light Industry of Science and Technology.

\section{References}

[1] W. Jiang, Z. Zheng, Y. Zhu, and Y. Li, "Demodulation for hydraulic pump fault signals based on local mean decomposition and improved adaptive multiscale morphology analysis," Mechanical Systems and Signal Processing, vol. 58, pp. 179-205, 2015.

[2] H. X. Chen, P. S. K. Chua, and G. H. Lim, "Adaptive wavelet transform for vibration signal modelling and application in fault diagnosis of water hydraulic motor," Mechanical Systems and Signal Processing, vol. 20, no. 8, pp. 2022-2045, 2006.

[3] M.-C. Pan and W.-C. Tsao, "Using appropriate IMFs for envelope analysis in multiple fault diagnosis of ball bearings," International Journal of Mechanical Sciences, vol. 69, pp. 114-124, 2013.

[4] T. Liu, J. Chen, and G.-M. Dong, "Rolling element bearing fault diagnosis based on frequency band entropy," Journal of Vibration and Shock, vol. 33, no. 1, pp. 77-80, 2014.

[5] F. Jiang, Z. Zhu, W. Li, G. Zhou, and G. Chen, "Fault identification of rotor-bearing system based on ensemble empirical mode decomposition and self-zero space projection analysis," Journal of Sound and Vibration, vol. 333, no. 14, pp. 3321-3331, 2014.

[6] F. Chaari, W. Bartelmus, R. Zimroz, T. Fakhfakh, and M. Haddar, "Gearbox vibration signal amplitude and frequency modulation," Shock and Vibration, vol. 19, no. 4, pp. 635-652, 2012.

[7] C. Peng, L. Bo, and X.-L. Xie, "Fault diagnosis method of rolling element bearings based on EEMD, measure-factor and fast kurtogram," Journal of Vibration and Shock, vol. 31, no. 20, pp. 143-146, 2012.

[8] Z. K. Peng, P. W. Tse, and F. L. Chu, "An improved HilbertHuang transform and its application in vibration signal analysis," Journal of Sound and Vibration, vol. 286, no. 1-2, pp. 187205, 2005.
[9] C. Wang, M. Gan, and C. Zhu, "Non-negative EMD manifold for feature extraction in machinery fault diagnosis," Measurement, vol. 70, pp. 188-202, 2015.

[10] W. Guo and P. W. Tse, "A novel signal compression method based on optimal ensemble empirical mode decomposition for bearing vibration signals," Journal of Sound and Vibration, vol. 332, no. 2, pp. 423-441, 2013.

[11] D. Boutana, M. Benidir, and B. Barkat, "On the selection of intrinsic mode function in EMD method: application on heart sound signal," in Proceedings of the 3rd International Symposium on Applied Sciences in Biomedical and Communication Technologies (ISABEL '10), pp. 1-5, IEEE, Rome, Italy, November 2010.

[12] K. S. Wang and P. S. Heyns, "An empirical re-sampling method on intrinsic mode function to deal with speed variation in machine fault diagnostics," Applied Soft Computing Journal, vol. 11, no. 8, pp. 5015-5027, 2011.

[13] Q. Wang, H. Chen, P. Chua, and L. G. Hian, "Fault detection of water hydraulic motor by demodulated vibration signal analysis with the hilbert transform and genetic algorithm," Journal of Testing and Evaluation, vol. 39, no. 2, 2011.

[14] B. Liang, S. Iwnicki, A. Ball, and A. E. Young, "Adaptive noise cancelling and time-frequency techniques for rail surface defect detection," Mechanical Systems and Signal Processing, vol. 54-55, pp. 41-51, 2015.

[15] N. E. Huang, Z. Shen, S. R. Long et al., "The empirical mode decomposition and the Hilbert spectrum for nonlinear and non-stationary time series analysis," Proceedings of the Royal Society of London. Series A: Mathematical, Physical and Engineering Sciences, vol. 454, no. 1971, pp. 903-995, 1998. 


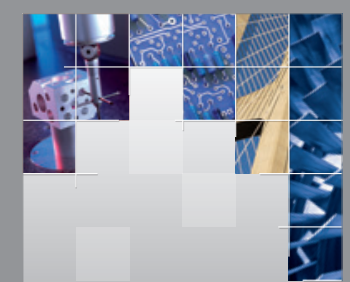

\section{Enfincering}
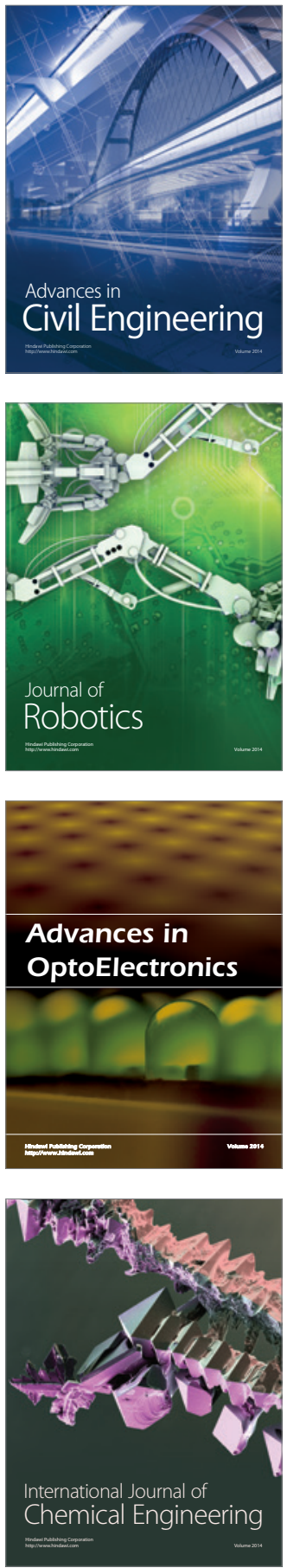

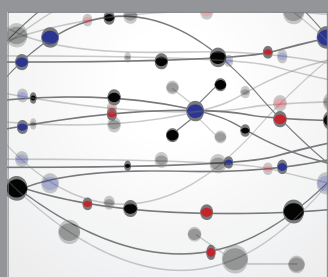

The Scientific World Journal

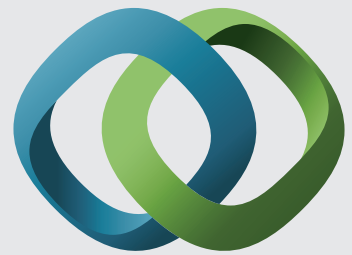

\section{Hindawi}

Submit your manuscripts at

http://www.hindawi.com
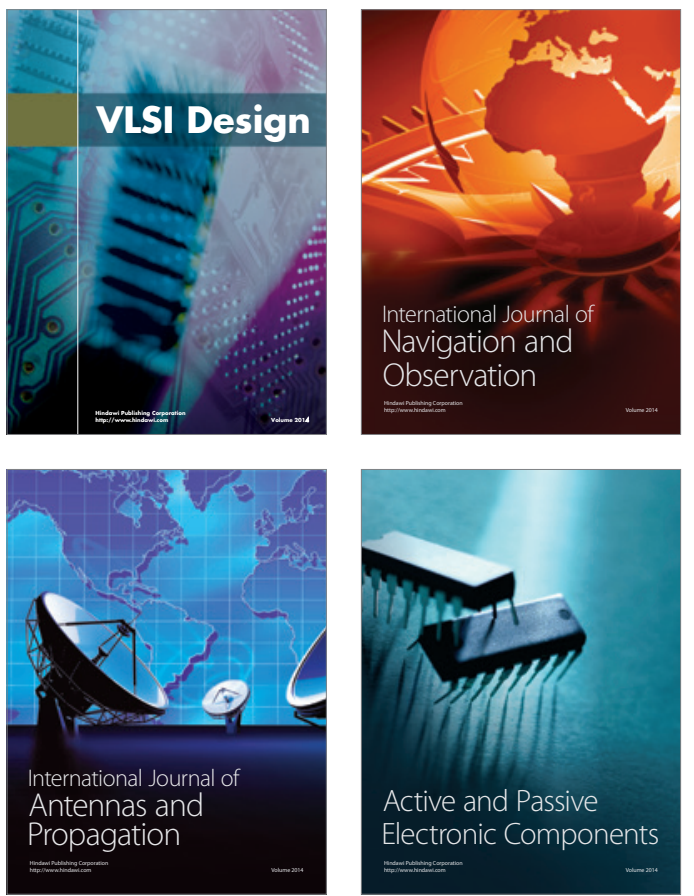
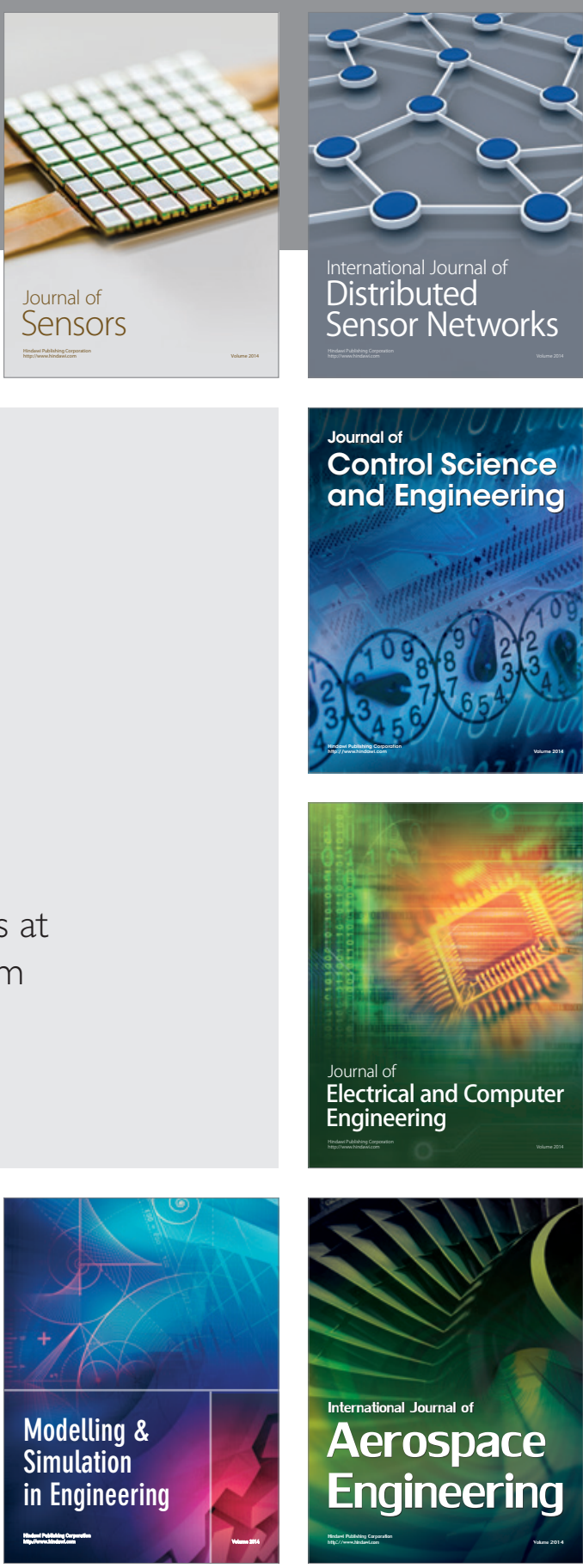

International Journal of

Distributed

Sensor Networks

Journal of

Control Science

and Engineering
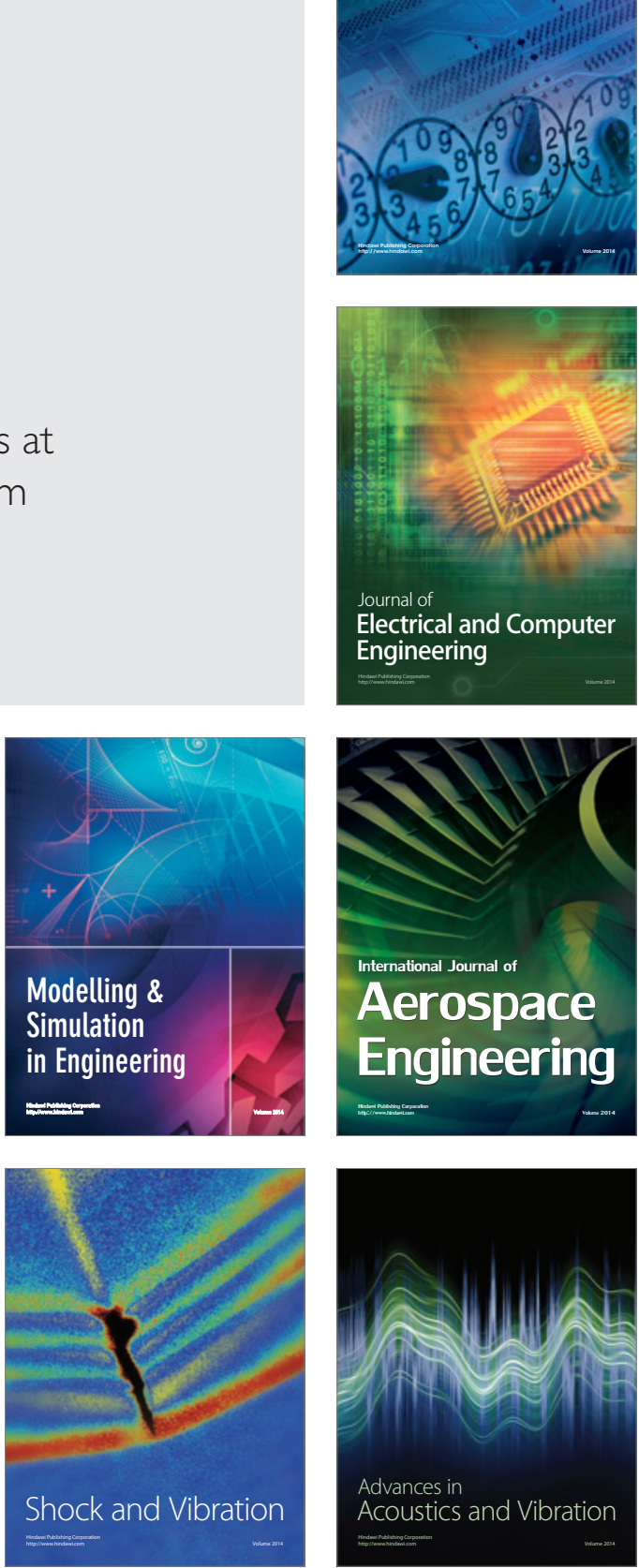